\title{
A New Method for Solving Second-Order Cone Eigenvalue Complementarity Problems
}

\author{
Samir Adly • Hadia Rammal
}

\begin{abstract}
In this paper, we study numerical methods for solving eigenvalue complementarity problems involving the product of second-order cones (or Lorentz cones). We reformulate such problem to find the roots of a semismooth function. An extension of the Lattice Projection Method (LPM) to solve the second-order cone eigenvalue complementarity problem is proposed. The LPM is compared to the semismooth Newton methods, associated to the Fischer-Burmeister and the natural residual functions. The performance profiles highlight the efficiency of the LPM. A globalization of these methods, based on the smoothing and regularization approaches, are discussed.
\end{abstract}

Keywords Lorentz cone - Second-order cone eigenvalue complementarity problem • Semismooth Newton method · Lattice Projection Method

\section{Introduction}

The theory of Complementarity Problems plays an important role in nonlinear optimization. The complementarity appears, for example, in the optimality conditions for constrained nonlinear programming or variational inequalities when the set of constraints is a closed and convex cone. The particular case of linear complementarity

S. Adly $(\bowtie) \cdot$ H. Rammal

XLIM UMR-CNRS 7252, Université de Limoges, 87060 Limoges, France

e-mail: samir.adly@unilim.fr

H. Rammal

e-mail: hadia.rammal@xlim.fr 
problems was widely discussed in the literature from both the theoretical and numerical point of view. More recently, the subject of eigenvalue complementarity problems has become one of the most well-established discipline in nonlinear optimization. It consists, for a given square matrix, in finding a scalar (eigenvalue) and nonzero vector (eigenvector) satisfying a complementarity condition over a closed and convex cone. When this cone coincides with the non-negative orthant, the problem is called Pareto eigenvalue complementarity problem. In this case, the spectrum is finite and its cardinality grows exponentially with the size of the matrix (see [1-3] for more details).

A wide variety of applications in Sciences and Engineering requires the resolution of eigenvalue complementarity problems such as the dynamic analysis of structural mechanical systems, vibro-acoustic systems, electrical circuit simulation, fluid dynamic, contact problem in mechanics (see, for instance, [4-8]). The development of robust and efficient algorithms for solving eigenvalue complementarity problems (EiCP, for short) has received more attention in recent years such as the spectral projected gradient algorithm [9], the semismooth Newton method [2], the scaling and projection algorithm [10] or the Path solver [11].

More recently, a wide range of applications in engineering design, transportation science, game theory, and economic equilibrium, can be formulated as optimization problems involving second-order cone constrained [12] (know also as the Lorentz cone). Equally important is the study of second-order cone complementarity problems (SOCCP, for short) since it contains a large class of problems such as, for instance, nonlinear complementarity problems and second-order cone programming problems. Fukushima et al. [13], Chen et al. [14,15] studied smooth and nonsmooth approaches. In [13], it has been shown that the natural residual and the Fischer-Burmeister functions can be extended to the SOCCP by means of Jordan Algebra.

There are presently certain theoretical properties and a large variety of computational methods for solving SOCCP. We cite, for example, interior-points methods, smoothing methods, SQP-type methods ([13-23]). Furthermore, the projection mapping onto second-order cone (SOC, for short) was studied by several authors. In $[14,15]$, it was proved that projection mapping onto SOC is a strongly semismooth function. Later, in 2005, Hayashi et al. [19] gave an explicit representation for the Jacobian of the projection onto SOC.

Motivated by these recent developments, we study throughout this paper, the second-order eigenvalue complementarity problems (SOCEiCP). We reformulate the SOCEiCP into a system of semismooth equations by using the second-order cone complementarity functions (SOCC-functions, for short), the most frequently used in the literature, namely, the min-function and the Fischer-Burmeister function. Our main goal in this paper is to introduce the extended Lattice Projection Method (LPM) for solving SOCEiCP. LPM has been first introduced in [1] to solve the Pareto eigenvalue complementarity problems. The second-order cone eigenvalue complementarity problems is considered to be one of the most difficult problems to solve, and this is due to the structure of the Lorentz spectrum which may be continuous (not finite and not countable). Therefore, compute and detect all the eigenvalues of this problem are not an easy task. As far as the authors know, this kind of technique for solving SOCE$\mathrm{iCP}$ has never been studied before. In addition, we study under which conditions the 
Jacobian matrix in the SNM algorithm (see Algorithm 1), at a solution, is nonsingular. LPM is then compared to the semismooth Newton methods: $\mathrm{SNM}_{\min }$ and $\mathrm{SNM}_{\mathrm{FB}}$, by using the performance profiles $[24,25]$ as a comparison tool. The numerical experiments highlight that the LPM solver is efficient for solving SOCEiCP.

This paper is organized as follows. In Sect. 2, we recall some background material on Euclidean Jordan Algebras and review the spectral factorization associated with a Lorentz cone. Moreover, we state a number of preliminary results for the projection mapping onto a second-order cone. In Sect. 3, we reformulate SOCEiCP into a system of semismooth equations. We extend, in Sect. 4, LPM to solve the SOCEiCP and give its B-subdifferential. In Sect. 5, we study the nonsingularity conditions of the Jacobian matrix, defined in the SNM algorithm. Then, we compare the LPM, in Sect. 6, with $\mathrm{SNM}_{\min }$ using the min-function and $\mathrm{SNM}_{\mathrm{FB}}$ via the Fischer-Burmeister function. In Sect. 7, we discuss a globalization of the three methods studied in the last sections by constructing appropriate merit functions. Finally, some concluding remarks are presented in Sect. 8 .

\section{Preliminaries}

In this section, we recall some background material and preliminary results on complementarity problems and Euclidean Jordan Algebras (see, e.g., [26,27]) as well as some definitions and properties of the $B$-subdifferential and (strong) semismoothness, which will be used in next sections.

We recall that the Pareto eigenvalue complementarity problem consists in finding a scalar $\lambda \in \mathbb{R}$ and a vector $x \in \mathbb{R}^{n} \backslash\{0\}$ such that

$$
x \geq 0, \lambda x-A x \geq 0 \text { and }\langle x, \lambda x-A x\rangle=0,
$$

where $A$ is real matrix of order $n$. The classical complementarity problem consists in finding $z \in \mathbb{R}^{n}$ such that

$$
z \geq 0, \quad F(z) \geq 0 \text { and }\langle z, F(z)\rangle=0,
$$

where $F: \mathbb{R}^{n} \rightarrow \mathbb{R}^{n}$ is a map. As mentioned in [28], the EiCP can be formulated as the following variational inequality (VI):

Find $x \in C=\left\{x \in \mathbb{R}_{+}^{n}:\langle e, x\rangle=1\right\}$ such that

$$
\langle F(x), y-x\rangle \geq 0, \quad \forall y \in C,
$$

where $F: \mathbb{R}^{n} \rightarrow \mathbb{R}^{n}$ is defined by

$$
F(x):=\left(\frac{x^{T} A x}{x^{T} x} I-A\right) x,
$$


$I$ is the identity matrix and $e$ is a column vector, whose components are all one. The second-order cone complementarity problem consists in finding $\zeta \in \mathbb{R}^{m}$ satisfying

$$
f(\zeta) \in \mathcal{K}, \quad g(\zeta) \in \mathcal{K}, \quad\langle f(\zeta), g(\zeta)\rangle=0
$$

where $f, g: \mathbb{R}^{m} \rightarrow \mathbb{R}^{n}$ are continuously differentiable mapping and $\mathcal{K}$ is the Cartesian product of second-order cones in $\mathbb{R}^{n}$, defined by

$$
\mathcal{K}:=\mathcal{K}^{n_{1}} \times \mathcal{K}^{n_{2}} \times \cdots \times \mathcal{K}^{n_{r}},
$$

and $n=n_{1}+n_{2}+\cdots+n_{r} \cdot \mathcal{K}^{n_{i}} \subset \mathbb{R}^{n_{i}}$ is the $n_{i}$-dimensional second-order cone, also called the Lorentz cone or ice cream cone, defined by

$$
\mathcal{K}^{n_{i}}:=\left\{x=\left(x_{1 i}, x_{2 i}\right) \in \mathbb{R} \times \mathbb{R}^{n_{i}-1}: x_{1 i} \geq\left\|x_{2 i}\right\|\right\}
$$

where $\|\cdot\|$ denotes the Euclidean norm defined by $\|x\|:=\sqrt{x^{T} x}$ for a vector $x \in \mathbb{R}^{n}$.

The cone $\mathcal{K}$ is self-dual, i.e., $\mathcal{K}^{+}=\mathcal{K}$, where $\mathcal{K}^{+}$is the dual cone of $\mathcal{K}$, defined by

$$
\mathcal{K}^{+}:=\left\{x \in \mathbb{R}^{n}:\langle x, y\rangle \geq 0, \forall y \in \mathcal{K}\right\}
$$

Corresponding to the Cartesian structure of $\mathcal{K}$, we write in (1) $f=\left(f_{1}, \ldots, f_{r}\right)$ and $g=\left(g_{1}, \ldots, g_{r}\right)$ with $f_{i}, g_{i}: \mathbb{R}^{m} \rightarrow \mathbb{R}^{n_{i}}$.

The second-order cone eigenvalue complementarity problem consists in finding a scalar $\lambda>0$ and a vector $x \in \mathbb{R}^{n} \backslash\{0\}$ satisfying

$$
x \in \mathcal{K}, \lambda x-A x \in \mathcal{K} \text { and }\langle x, \lambda x-A x\rangle=0 .
$$

The scalar $\lambda$ is called the Lorentz eigenvalue of the real matrix $A \in \mathbb{M}_{n}(\mathbb{R})$. From (1), it is sufficient to take

$$
m=n+1, \zeta=(x, \lambda), f(\zeta)=f(x, \lambda)=x \text { and } g(\zeta)=g(x, \lambda)=\lambda x-A x
$$

Then, (1) reduces to (3).

For two $n$-dimensional vectors $x=\left(x_{1}, x_{2}\right)$ and $y=\left(y_{1}, y_{2}\right) \in \mathbb{R} \times \mathbb{R}^{n-1}$, we define their Jordan product as

$$
x \circ y:=\left(x^{T} y, x_{1} y_{2}+y_{1} x_{2}\right)
$$

An important character of Jordan Algebra is its eigen-decomposition, also called the spectral factorization with respect to the second-order cone $\mathcal{K}^{n}$. For more details, see, e.g., $[13,26]$.

For any vector $x=\left(x_{1}, x_{2}\right) \in \mathbb{R} \times \mathbb{R}^{n-1}$, its spectral factorization is defined as

$$
x:=\lambda_{1}(x) u^{(1)}(x)+\lambda_{2}(x) u^{(2)}(x),
$$


where $\lambda_{1}, \lambda_{2} \in \mathbb{R}$ and $u^{(1)}, u^{(2)} \in \mathbb{R}^{n}$ are the spectral values and the associated spectral vectors of $x$, respectively, given by

$$
\lambda_{i}(x)=x_{1}+(-1)^{i}\left\|x_{2}\right\|, \quad i=1,2,
$$

and

$$
u^{(i)}(x)=\left\{\begin{array}{lr}
\frac{1}{2}\left(1,(-1)^{i} \frac{x_{2}}{\left\|x_{2}\right\|}\right), & \text { if } x_{2} \neq 0, \\
\frac{1}{2}\left(1,(-1)^{i} w\right), & \text { if } x_{2}=0,2,
\end{array}\right.
$$

with $w \in \mathbb{R}^{n-1}$, such that $\|w\|=1$.

For the proof of (5), (6) and (7), see, e.g., [29].

We recall now some definitions and properties of the $B$-subdifferential and (strong) semismoothness, which will be used later.

Let $H: \mathbb{R}^{n} \rightarrow \mathbb{R}^{m}$ be a locally Lipschitz function. Then, the set

$$
\partial_{B} H(z):=\left\{M \in \mathbb{R}^{m \times n}: \exists\left(z^{k}\right) \subseteq D_{H}: z^{k} \rightarrow z \text { and } \lim _{k \rightarrow+\infty} \nabla H\left(z^{k}\right)=M\right\}
$$

where $D_{H}:=\left\{z \in \mathbb{R}^{n}: H\right.$ is differentiable at $\left.z\right\}$, is nonempty and is called the $B$ subdifferential of $H$. The Clarke subdifferential [30] of $H$ is defined by the convex hull

$$
\partial H(z):=\operatorname{conv} \partial_{B} H(z) \text {. }
$$

A vector $z$ is called a $B D$-regular for $H$ iff all matrices in the $B$-subdifferential $\partial_{B} H(z)$ are non-singular.

By using the subdifferential and the directional derivative (denoted by $H^{\prime}(z ; d)$ ), we define semismoothness and strong semismoothness, which are first introduced by Mifflin [31] and extended later by Qi and Sun [32].

A directionally differentiable and locally Lipschitz function $H: \mathbb{R}^{n} \rightarrow \mathbb{R}^{m}$ is said to be semismooth at $x$, iff

$$
M d-H^{\prime}(x ; d)=o(\|d\|),
$$

for all $M \in \partial H(z+d)$ and $d \rightarrow 0$. Moreover, if $o(\|d\|)$ can be replaced by $O\left(\|d\|^{2}\right)$, then the function $H$ is said to be strongly semismooth.

Next, we recall some properties of the projection of any $z \in \mathbb{R}^{n}$ onto a second-order cone. 


\subsection{Projection onto (SOC)}

For $z \in \mathbb{R}^{n}$, the projection of $z$ onto the second-order cone $\mathcal{K}^{n}$ is defined by

$$
P_{\mathcal{K}^{n}}(z):=\arg \min _{z^{\prime} \in \mathcal{K}^{n}}\left\|z^{\prime}-z\right\|
$$

It can be written explicitly as

$$
P_{\mathcal{K}^{n}}(z)=\max \left\{0, \lambda_{1}\right\} u^{1}+\max \left\{0, \lambda_{2}\right\} u^{2},
$$

where $\lambda_{1}, \lambda_{2}$ and $u^{1}, u^{2}$ are defined by (5) and (6) [13, Proposition 3.3]. Hence, we get

$$
P_{\mathcal{K}^{n}}(z)= \begin{cases}z & \left(\lambda_{1} \geq 0, \lambda_{2} \geq 0\right), \\ \frac{1}{2}\left(z_{1}+\left\|z_{2}\right\|\right)\left(1, \frac{z_{2}}{\left\|z_{2}\right\|}\right) & \left(\lambda_{1}<0, \lambda_{2}>0\right), \\ 0 & \left(\lambda_{1} \leq 0, \lambda_{2} \leq 0\right)\end{cases}
$$

which is strongly semismooth at any $z \in \mathbb{R}^{n}[14]$.

Lemma 2.1 [33] The projection mapping $P_{\mathcal{K}^{n}}$ is differentiable at a point $z=$ $\left(z_{1}, z_{2}\right) \in \mathbb{R} \times \mathbb{R}^{n-1}$ if and only if $z_{1} \neq\left\|z_{2}\right\|$ holds. In fact, the projection mapping is continuously differentiable at every $z$ such that $z_{1} \neq\left\|z_{2}\right\|$, i.e., $z \notin \operatorname{bd}\left(\mathcal{K}^{n}\right)$, where $b d\left(\mathcal{K}^{n}\right)$ is the boundary of $\mathcal{K}^{n}$ defined by

$$
\operatorname{bd}\left(\mathcal{K}^{n}\right):=\left\{z=\left(z_{1}, z_{2}\right) \in \mathbb{R} \times \mathbb{R}^{n-1}: z_{1}=\left\|z_{2}\right\|\right\}
$$

Lemma 2.2 For any $z=\left(z_{1}, z_{2}\right) \in \mathbb{R} \times \mathbb{R}^{n-1}$, an element $V$ of the $B$-subdifferential $\partial_{B} P_{\mathcal{K}^{n}}(z)$ has the following representation:

$$
\begin{array}{lll}
V=I & \left(\lambda_{1}>0, \lambda_{2}>0\right), \text { i.e., } z \in \operatorname{int}\left(\mathcal{K}^{n}\right), \\
V=\frac{\lambda_{2}}{\lambda_{2}-\lambda_{1}} I+Z & \left(\lambda_{1}<0, \lambda_{2}>0\right), \text { i.e., } z \in \mathbb{R}^{n} \backslash\left(\mathcal{K}^{n} \cup-\mathcal{K}^{n}\right), \\
V=0_{n} & \left(\lambda_{1}<0, \lambda_{2}<0\right), \text { i.e., } z \in-\operatorname{int}\left(\mathcal{K}^{n}\right), \\
V \in\{I, I+Z\} & \left(\lambda_{1}=0, \lambda_{2}>0\right), \text { i.e., } z \in \operatorname{bd}\left(\mathcal{K}^{n}\right) \backslash\{0\}, \\
V \in\left\{0_{n}, Z\right\} & \left(\lambda_{1}<0, \lambda_{2}=0\right), \text { i.e., } z \in-\operatorname{bd}\left(\mathcal{K}^{n}\right) \backslash\{0\}, \\
V=0_{n} \text { or } V=I \text { or } V \in S & \left(\lambda_{1}=0, \lambda_{2}=0\right), \text { i.e., } z \in\{0\},
\end{array}
$$

where

$$
\begin{aligned}
\left(r_{1}, r_{2}\right) & :=\frac{\left(z_{1}, z_{2}\right)}{\left\|z_{2}\right\|}, \quad Z=\frac{1}{2}\left(\begin{array}{ll}
-r_{1} & r_{2}^{T} \\
r_{2} & -r_{1} r_{2} r_{2}^{T}
\end{array}\right) \quad \text { and } \\
S & :=\left\{\frac{1}{2}(1+\beta) I+\frac{1}{2}\left(\begin{array}{ll}
-\beta & \omega^{T} \\
\omega & -\beta \omega \omega^{T}
\end{array}\right):-1 \leq \beta \leq 1,\|\omega\|=1\right\} .
\end{aligned}
$$

Proof We can find a similar representation for the elements of $\partial_{B} P_{\mathcal{K}^{n}}(z)$ and those of the Clarke subdifferential $\partial P_{\mathcal{K}^{n}}(z)$ in [15, Lemma 4], [19, Proposition 4.3], [33, Lemma 2.5] and [34, Lemma 14]. 


\section{Second-Order Eigenvalue Complementarity Problem}

Throughout this section, we deal with the second-order cone eigenvalue complementarity problem defined in (3), which consists in finding a scalar $\lambda>0$ and a vector $x \in \mathbb{R}^{n} \backslash\{0\}$ satisfying

$$
x \in \mathcal{K}, \lambda x-A x \in \mathcal{K},\langle x, \lambda x-A x\rangle=0 .
$$

The set

$$
\sigma(A, \mathcal{K}):=\{\lambda>0:(\lambda, x) \text { solves (3) for some } x \in \mathcal{K} \backslash\{0\}\}
$$

is said to be the Lorentz spectrum of $A$. The component $\lambda$ is called a Lorentz eigenvalue of $A$, while the component $x$ is called a Lorentz eigenvector of $A$. The eigenvalue complementarity problem in the the case of the second-order cone has recently been studied. For this reason, the number of publications is rather limited (see, for instance, [35]).

In [35], Seeger and Torki studied the main properties and the structure of the Lorentz spectral mapping $\sigma(., \mathcal{K})$. They recovered $\sigma(A, \mathcal{K})$, defined in $(12)$, by joining two pieces and treated each one:

$$
\sigma(A, \mathcal{K})=\sigma_{\text {int }}(A, \mathcal{K}) \cup \sigma_{\text {bd }}(A, \mathcal{K}),
$$

where $\sigma_{\text {int }}(A, \mathcal{K})$ (respectively, $\sigma_{\text {bd }}(A, \mathcal{K})$ ) is the set of all eigenvalues $\lambda$ of $A$ associated with eigenvectors in the interior of $\mathcal{K}$ (respectively, in the boundary of $\mathcal{K}$ ).

Moreover, they characterized the elements in the Lorentz spectrum and provided a valuable information on their number. They announced that $\sigma(A, \mathcal{K})$ is not necessarily finite, but it can always be written as union of finitely many (at most $5 n-4$ ) mutually disjoint connected sets [35, Theorem 4.1 and Corollary 4.4] (see examples 3.1 and 3.2).

Furthermore, they showed the classes of matrices over which the Lorentz cone can produce only a finite number of eigenvalues.

Proposition 3.1 [35] In each of the following cases, the real matrix

$A \in \mathbb{R}^{n \times n}$ has a finite number of Lorentz eigenvalues:

(a) A is symmetric,

(b) the leading $(n-1) \times(n-1)$ principal submatrix of $A$ has no (real) eigenvalues with geometric multiplicity $\geq 2$.

In what follows, we give some examples of discrete and continuous solutions.

Example 3.1 Consider the following matrix

$$
A=\left[\begin{array}{lllll}
3 & 0 & 0 & 0 & 0 \\
0 & 5 & 0 & 0 & 0 \\
0 & 0 & 5 & 0 & 0 \\
0 & 0 & 0 & 7 & 0 \\
0 & 0 & 0 & 0 & 7
\end{array}\right]
$$


$A$ has a discrete Lorentz spectrum given by

$$
\sigma(A, \mathcal{K})=\{3,4,5\}
$$

Example 3.2 Let

$$
B=\left[\begin{array}{lllll}
3 & \frac{2}{3} & 0 & \frac{2}{3} & 0 \\
0 & 5 & 0 & 0 & 0 \\
0 & 0 & 5 & 0 & 0 \\
0 & 0 & 0 & 7 & 0 \\
0 & 0 & 0 & 0 & 7
\end{array}\right]
$$

We have

$$
\sigma(B, \mathcal{K})=\{3\} \cup[4.0009,4.3333] \cup[5.0016,5.3333]
$$

which is a continuous Lorentz spectrum of $B$.

As a first step toward a reformulation of the SOCEiCP as a system of equations, we write

$$
x \in \mathcal{K}, y \in \mathcal{K},\langle x, y\rangle=0, \lambda x-A x-y=0,\left\langle\mathbf{1}_{n}, x\right\rangle-1=0,
$$

where $\mathbf{1}_{n}$ is a vector of ones. The normalization equation (the last equation in (13)) is used to ensure that $x$ is a nonzero vector.

Without any loss of generality, we can suppose that $\lambda$ is strictly positive. If the matrix $A$ has a negative eigenvalue $\lambda<0$, we set $\tilde{A}=A+\mu \mathbf{I}_{n}$, where $\mu>0$ is large enough. It is easy then to observe that the eigenvalues of $\tilde{A}$ are strictly positive.

The SOCEiCP can be reformulated as the following nonsmooth system of equations

$$
\Phi(z)=\Phi(x, y, \lambda):=\left[\begin{array}{l}
\varphi^{1}\left(x_{1}, y_{1}\right) \\
\vdots \\
\varphi^{r}\left(x_{r}, y_{r}\right) \\
\lambda x-A x-y \\
\left\langle\mathbf{1}_{n}, x\right\rangle-1
\end{array}\right]=0
$$

where $\Phi: \mathbb{R}^{n} \times \mathbb{R}^{n} \times \mathbb{R} \rightarrow \mathbb{R}^{2 n+1}$ and $\varphi^{i}: \mathbb{R}^{n_{i}} \times \mathbb{R}^{n_{i}} \rightarrow \mathbb{R}^{n_{i}}$ is a second-order cone complementarity function (SOCC-function) associated with the cone $\mathcal{K}^{n_{i}}\left(n_{i} \geq 1\right)$ on Euclidean Jordan Algebras, i.e.,

$$
\varphi^{i}\left(x_{i}, y_{i}\right)=0 \Longleftrightarrow x_{i} \in \mathcal{K}^{n_{i}}, y_{i} \in \mathcal{K}^{n_{i}}, x_{i}^{T} y_{i}=0 .
$$

In this paper, we focus on the natural residual function, also called, the minfunction, denoted by $\varphi_{\mathrm{min}}^{\mathrm{soc}}$, and the Fischer-Burmeister function, denoted by $\varphi_{\mathrm{FB}}^{\mathrm{soc}}$, defined, respectively, by 


$$
\begin{aligned}
\varphi_{\min }^{\mathrm{soc}}(x, y) & :=x-P_{\mathcal{K}^{n}}(x-y), \\
\varphi_{\mathrm{FB}}^{\mathrm{soc}}(x, y) & :=x+y-\left(x^{2}+y^{2}\right)^{1 / 2} .
\end{aligned}
$$

Note that the $\varphi_{\mathrm{FB}}^{\mathrm{SOc}}$ function defined above was proposed by Gowda et al. in [36]. Xiu and al. established also the definition of $\varphi_{\mathrm{FB}}^{\mathrm{soc}}$ and proved that it is Lipschitz continuous in [37]. Furthermore, Sun and Sun in [21] showed that it is strongly semismooth everywhere, and so is the $\varphi_{\min }^{\mathrm{soc}}$ function (see [14] for more details).

In what follows, we give the B-subdifferential of $\Phi$ in two cases. The first case is defined when the SOCC-function is given by the $\varphi_{\min }^{\mathrm{soc}}$ function defined in (16), and the second one is related to the $\varphi_{\mathrm{FB}}^{\mathrm{Soc}}$ function, defined in (17).

Lemma 3.1 The function $\Phi_{\min }: \mathbb{R}^{n} \times \mathbb{R}^{n} \times \mathbb{R} \rightarrow \mathbb{R}^{2 n+1}$, defined in (14) with $\varphi$ being the $\varphi_{\mathrm{min}}^{\mathrm{soc}}$ function, is semismooth. Moreover, each element $H_{\min }$ of the $B$-subdifferential of $\Phi_{\min }$ at $z=(x, y, \lambda)$ is given by

$$
H_{\min }=\left[\begin{array}{lll}
I-V & V & 0 \\
\lambda I-A & -I & x \\
\mathbf{1}_{n}^{T} & 0 & 0
\end{array}\right]
$$

where $V \in \partial_{B} P_{\mathcal{K}^{n}}(x-y)$.

Taking into account the proposition 3.1 in [38], we have the following.

Given a vector $(x, y) \in \mathbb{R}^{n} \times \mathbb{R}^{n}$, then each element $V_{\mathrm{FB}}$ in $\partial_{B} \varphi_{\mathrm{FB}}^{\text {soc }}(x, y)$ is given by

$$
V_{\mathrm{FB}}=\left[\begin{array}{ll}
I-V_{x} & I-V_{y}
\end{array}\right]
$$

with $V_{x}$ and $V_{y}$ having the following representation:

(i) If $x^{2}+y^{2} \in \operatorname{int}\left(\mathcal{K}^{n}\right)$, then $V_{x}=L_{w}^{-1} L_{x}$ and $V_{y}=L_{w}^{-1} L_{y}$, where $w:=\left(x^{2}+y^{2}\right)^{1 / 2}$, and for $x \in \mathcal{K}^{n}, L_{x}=\left[\begin{array}{cc}x_{1} & x_{2}^{T} \\ x_{2} & x_{1} I\end{array}\right]$. If $x \in \operatorname{int}\left(\mathcal{K}^{n}\right)$, where

$$
\operatorname{int}\left(\mathcal{K}^{n}\right):=\left\{x=\left(x_{1}, x_{2}\right) \in \mathbb{R} \times \mathbb{R}^{n-1}: x_{1}>\left\|x_{2}\right\|\right\}
$$

then,

$$
L_{x}^{-1}=\frac{1}{\operatorname{det}(x)}\left[\begin{array}{cc}
x_{1} & -x_{2}^{T} \\
-x_{2} & \frac{\operatorname{det}(x)}{x_{1}} I+\frac{x_{2} x_{2}^{T}}{x_{1}}
\end{array}\right]
$$

where $\operatorname{det}(x):=x_{1}^{2}-\left\|x_{2}\right\|^{2}$ denotes the determinant of $x$. 
(ii) If $x^{2}+y^{2} \in \operatorname{bd}\left(\mathcal{K}^{n}\right)$ and $(x, y) \neq(0,0)$, then

$$
\begin{aligned}
& V_{x} \in\left\{\frac{1}{2 \sqrt{2 l_{1}}}\left(\begin{array}{ll}
1 & \bar{l}_{2}^{T} \\
\overline{l_{2}} & 4 I-3 \overline{l_{2}} \bar{l}_{2}^{T}
\end{array}\right) L_{x}+\frac{1}{2}\left(\begin{array}{l}
1 \\
-\overline{l_{2}}
\end{array}\right) u^{T}\right\} \\
& V_{y} \in\left\{\frac{1}{2 \sqrt{2 l_{1}}}\left(\begin{array}{ll}
1 & \bar{l}_{2}^{T} \\
\overline{l_{2}} 4 I-3 \overline{l_{2}} \bar{l}^{T}
\end{array}\right) L_{y}+\frac{1}{2}\left(\begin{array}{l}
1 \\
-\overline{l_{2}}
\end{array}\right) v^{T}\right\},
\end{aligned}
$$

where $u=\left(u_{1}, u_{2}\right), v=\left(v_{1}, v_{2}\right) \in \mathbb{R} \times \mathbb{R}^{n-1}$ satisfying $\left|u_{1}\right| \leq\left\|u_{2}\right\| \leq 1$ and $\left|v_{1}\right| \leq\left\|v_{2}\right\| \leq 1$, where $l=\left(l_{1}, l_{2}\right):=x^{2}+y^{2}$ and $\overline{l_{2}}=l_{2} /\left\|l_{2}\right\|$.

(iii) If $(x, y)=(0,0)$, then $V_{x} \in\left\{L_{\tilde{x}}\right\}, V_{y} \in\left\{L_{\tilde{y}}\right\}$ for some $\tilde{x}, \tilde{y}$ with $\|\tilde{x}\|^{2}+\|\tilde{y}\|^{2}=1$, or

$$
\begin{aligned}
& V_{x} \in\left\{\frac{1}{2}\left(\begin{array}{l}
1 \\
\overline{l_{2}}
\end{array}\right) \xi^{T}+\frac{1}{2}\left(\begin{array}{l}
1 \\
-\overline{l_{2}}
\end{array}\right) u^{T}+2\left(\begin{array}{ll}
0 & 0 \\
\left(I-\overline{l_{2}} \bar{l}_{2}^{T}\right) s_{2}\left(I-\overline{l_{2}} \bar{l}_{2}^{T}\right) s_{1}
\end{array}\right)\right\} \\
& V_{y} \in\left\{\frac{1}{2}\left(\begin{array}{l}
1 \\
\overline{l_{2}}
\end{array}\right) \eta^{T}+\frac{1}{2}\left(\begin{array}{l}
1 \\
-\overline{l_{2}}
\end{array}\right) v^{T}+2\left(\begin{array}{ll}
0 & 0 \\
\left(I-\overline{l_{2}} \bar{l}_{2}^{T}\right) l_{2} & \left(I-\overline{l_{2}} \bar{l}_{2}^{T}\right) l_{1}
\end{array}\right)\right\},
\end{aligned}
$$

where $u=\left(u_{1}, u_{2}\right), v=\left(v_{1}, v_{2}\right), \xi=\left(\xi_{1}, \xi_{2}\right), \eta=\left(\eta_{1}, \eta_{2}\right) \in \mathbb{R} \times \mathbb{R}^{n-1}$ satisfying $\left|u_{1}\right| \leq\left\|u_{2}\right\| \leq 1,\left|v_{1}\right| \leq\left\|v_{2}\right\| \leq 1,\left|\xi_{1}\right| \leq\left\|\xi_{2}\right\| \leq 1$ and

$\left|\eta_{1}\right| \leq\left\|\eta_{2}\right\| \leq 1$, where $\bar{l}_{2} \in \mathbb{R}^{n-1}$ such that $\left\|\bar{l}_{2}\right\|=1$, and $s=\left(s_{1}, s_{2}\right)$, $l=\left(\overline{l_{1}}, l_{2}\right) \in \overline{\mathbb{R}} \times \mathbb{R}^{n-1}$ satisfying $\|s\|^{2}+\|l\|^{2} \leq 1 / 2$.

For more details, see [38]. Therefore, we get the following lemma.

Lemma 3.2 The function $\Phi_{\mathrm{FB}}: \mathbb{R}^{n} \times \mathbb{R}^{n} \times \mathbb{R} \rightarrow \mathbb{R}^{2 n+1}$, defined in (14) with $\varphi$ being the $\varphi_{\mathrm{FB}}^{\mathrm{soc}}$ function, is semismooth. Moreover, each element $H_{\mathrm{FB}}$ in $\partial_{B} \Phi_{\mathrm{FB}}(z)$ is given by

$$
H_{\mathrm{FB}}=\left[\begin{array}{lll}
I-V_{x} & I-V_{y} & 0 \\
\lambda I-A & -I & x \\
\mathbf{1}_{n}^{T} & 0 & 0
\end{array}\right]
$$

where $V_{x}$ and $V_{y}$ are given by the above equations.

\section{The Lattice Projection Method (LPM)}

In this section, we generalize the LPM given in [1] to solve SOCEiCP, which leads us to solve a nonlinear and nonsmooth system of $(2 n+1)$ equations involving the same number of variables. The originality of this formulation is that it is not based on the complementarity approach.

Lemma 4.1 The SOCEiCP, with $\lambda>0$, consists in finding the roots of the following nonlinear and nonsmooth function $h: \mathbb{R}^{n} \times \mathbb{R} \rightarrow \mathbb{R}^{n},(x, \lambda) \mapsto h(x, \lambda)$, defined by

$$
h(x, \lambda):=P_{\mathcal{K}}(A x)-\lambda x .
$$


Proof Suppose that $\lambda>0$ and $x \in \mathcal{K}$. By the definition of the normal cone, we have

$$
\mathcal{K} \ni x \perp \lambda x-A x \in \mathcal{K} \Longleftrightarrow A x-\lambda x \in N_{\mathcal{K}}(x) .
$$

Therefore SOCEiCP is equivalent to

$$
x=P_{\mathcal{K}}\left(\frac{1}{\lambda} A x\right)
$$

Hence,

$$
P_{\mathcal{K}}(A x)=\lambda x
$$

In short, we are led to solve the following system of $(2 n+1)$ equations

$$
\begin{aligned}
P_{\mathcal{K}}(\tilde{y})-\lambda x & =0, \\
A x-\tilde{y} & =0, \\
\left\langle\mathbf{1}_{n}, x\right\rangle-1 & =0 .
\end{aligned}
$$

Remark 4.1 Lemma 4.1 showed that

$$
\mathcal{K} \ni x \perp(\lambda x-A x) \in \mathcal{K} \Longleftrightarrow P_{\mathcal{K}}(A x)=\lambda x,
$$

which means that the SOCEiCP is equivalent to solve the nonlinear eigenvalue problem

$$
\left(P_{\mathcal{K}} \circ A\right)(x)=\lambda x
$$

The SOCEiCP can be written equivalently to the following nonsmooth system of equations:

$$
\Phi_{\mathrm{LPM}}(\tilde{z}):=\left[\begin{array}{l}
P_{\mathcal{K}}(\tilde{y})-\lambda x \\
A x-\tilde{y} \\
<\mathbf{1}_{n}, x>-1
\end{array}\right]=0,
$$

where $\Phi_{\mathrm{LPM}}: \mathbb{R}^{n} \times \mathbb{R}^{n} \times \mathbb{R} \rightarrow \mathbb{R}^{2 n+1}, \tilde{z}=(x, \tilde{y}, \lambda) \mapsto \Phi_{\mathrm{LPM}}(\tilde{z})$.

Remark 4.2 We note that the solutions of the nonlinear equation (22) do not satisfy necessarily $\lambda>0$. Maintaining the non-negativity of $\lambda$ in the algorithm is not an easy task. If the matrix $A$ has a nonpositive eigenvalue $\lambda<0$, then we set $\tilde{A}=A+\mu I_{n}$ with $\mu>0$ and large enough. It is easy to see in this case that the spectrum $\sigma(\tilde{A}) \subset \mathbb{R}_{+}^{*}$.

The Algorithm 1 presented in the following part is used to solve (22). This method will be called LPM.

The following lemma gives a brief description of the representation of the Bsubdifferential of $\Phi_{\text {LPM }}$. 
Lemma 4.2 The function $\Phi_{\mathrm{LPM}}: \mathbb{R}^{n} \times \mathbb{R}^{n} \times \mathbb{R} \rightarrow \mathbb{R}^{2 n+1}$, defined in (22), is semismooth. Moreover, each element $\tilde{H}$ of the $B$-subdifferential of $\Phi_{\mathrm{LPM}}$ at $\tilde{z}=(x, \tilde{y}, \lambda)$ is given by

$$
\tilde{H}=\left[\begin{array}{lll}
-\lambda I & \tilde{V} & -x \\
A & -I & 0 \\
\mathbf{1}_{n}^{T} & 0 & 0
\end{array}\right]
$$

where $\tilde{V} \in \partial_{B} P_{\mathcal{K}}(\tilde{y})$.

Proof Chen et al. [14] showed that $P_{\mathcal{K}}(\cdot)$ is strongly semismooth, so that $\Phi_{\mathrm{LPM}}$ is semismooth. The computation of the B-subdifferential of $\Phi_{\mathrm{LPM}}$ offers no difficulty and we get obviously the representation formula (23).

The algorithm we are about to describe is the Semismooth Newton Method SNM for solving SOCEiCP.

\section{Algorithm 1 (Semismooth Newton Method SNM)}

Initialization. Choose an initial point $z^{0}$ and set $k=0$.

Iteration. ${ }^{1}$ One has a current point $z^{k}$. Choose $M^{k} \in \partial_{B} \Phi\left(z^{k}\right)$ and compute $h^{k}$ by solving the linear system

$$
M^{k} h^{k}=-\Phi\left(z^{k}\right) .
$$

Then, set $z^{k+1}=z^{k}+h^{k}$ and $k=k+1$,

where $\Phi$ is either $\Phi_{\mathrm{LPM}}$ or $\Phi_{\min }$ or $\Phi_{\mathrm{FB}}$. To ensure that the linear system (24) admits a unique solution, the matrix $M^{k}$ must be nonsingular. Furthermore, the following theorem gives some conditions to ensure the local convergence of the above algorithm [32].

Theorem 4.1 Let $\bar{z}$ be a zero of the function $\Phi$. Suppose the following

(i) $\Phi$ is semismooth (resp. strongly semismooth) at $\bar{z}$;

(ii) all matrices $M^{k} \in \partial_{B} \Phi(\bar{z})$ are nonsingular. Then, there exists a neighborhood $V$ of $\bar{z}$ such that the $S N M$, initialized at any $z^{0} \in V$, generates a sequence $\left(z^{k}\right)_{k \in \mathbb{N}}$ that converges superlinearly (resp. quadratically) to $\bar{z}$.

\section{Nonsingularity Conditions}

We study, in this section, under which conditions the Jacobain matrix, defined in (24) of the Algorithm 1, is nonsingular at a solution. More precisely, we study the nonsingularity conditions of the elements of the B-subdifferential of $\Phi\left(z^{*}\right)$ and $\Phi_{\mathrm{LPM}}\left(\tilde{z}^{*}\right)$ defined, respectively, in (14) and (22). Firstly, we give some notations, which will be used later.The index set $\{1,2, \ldots, n\}$ will be abbreviated by the capital letter $I$.

\footnotetext{
$\overline{{ }^{1} \text { As stopping criteria, we use } \|} \Phi\left(z^{k}\right) \|<10^{-8}$.
} 
If $M=\left(m_{i j}\right) \in \mathbb{M}_{n}(\mathbb{R})$ is a matrix and $\mathcal{J}, \mathcal{K} \subseteq I$, then $M_{\mathcal{J K}}$ denotes the submatrix in $\mathbb{M}_{|\mathcal{J}|,|\mathcal{K}|}(\mathbb{R})$ with elements $m_{i j}, i \in \mathcal{J}, j \in \mathcal{K}$.

We introduce the following function $F$ of class $C^{1}$

$$
\begin{aligned}
& F: \mathbb{R}^{n} \times \mathbb{R}^{n} \times \mathbb{R} \rightarrow \mathbb{R}^{2 n+1}, z=(x, y, \lambda) \mapsto F(z) \text { given by } \\
& F(z)=\left[\begin{array}{l}
y \\
\lambda x-A x-y \\
\left\langle\mathbf{1}_{n}, x\right\rangle-1
\end{array}\right] .
\end{aligned}
$$

Proposition 5.1 Any $H \in \partial_{B} \Phi(z)$, with $\varphi$ being one of the SOCC-functions $\varphi_{\min }^{\mathrm{soc}}$ or $\varphi_{\mathrm{FB}}^{\mathrm{SOc}}$, can be written in the form

$$
H=D_{a}+D_{b} F^{\prime}(z)
$$

where $D_{a}, D_{b} \in \mathbb{M}_{2 n+1}(\mathbb{R})$ are positive semidefinite diagonal matrices, defined by

$$
D_{a}=\left[\begin{array}{lll}
I-V & 0 & 0 \\
0 & 0 & 0 \\
0 & 0 & 0
\end{array}\right] \text { and } D_{b}=\left[\begin{array}{lll}
V & 0 & 0 \\
0 & I & 0 \\
0 & 0 & 1
\end{array}\right]
$$

in the case where $\varphi(x, y)=\varphi_{\min }^{\mathrm{soc}}(x, y)$.

$$
D_{a}=\left[\begin{array}{lll}
I-L_{w}^{-1} L_{x} & 0 & 0 \\
0 & 0 & 0 \\
0 & 0 & 0
\end{array}\right] \text { and } D_{b}=\left[\begin{array}{lll}
I-L_{w}^{-1} L_{y} & 0 & 0 \\
0 & I & 0 \\
0 & 0 & 1
\end{array}\right]
$$

in the case where $\varphi(x, y)=\varphi_{\mathrm{FB}}^{\mathrm{soc}}(x, y)$ and $x^{2}+y^{2} \in \operatorname{int}\left(\mathcal{K}^{n}\right)$.

Proof If $H \in \partial_{B} \Phi_{\min }(z)$, then by Lemma 3.1, we have

$$
H=H_{\min }=\left[\begin{array}{lll}
I-V & V & 0 \\
\lambda I-A & -I & x \\
\mathbf{1}_{n}^{T} & 0 & 0
\end{array}\right] .
$$

If $H \in \partial_{B} \Phi_{\mathrm{FB}}(z)$, then using Lemma 3.2, we get

$$
H=H_{\mathrm{FB}}=\left[\begin{array}{lll}
I-L_{w}^{-1} L_{x} & I-L_{w}^{-1} L_{y} & 0 \\
\lambda I-A & -I & x \\
\mathbf{1}_{n}^{T} & 0 & 0
\end{array}\right]
$$

On the other hand, we have

$$
F^{\prime}(z)=\left[\begin{array}{lll}
0 & I & 0 \\
\lambda I-A & -I & x \\
\mathbf{1}_{n}^{T} & 0 & 0
\end{array}\right]
$$

Therefore, from these equalities and using [39], we get the desired result. 
Now let $z^{*}=\left(x^{*}, y^{*}, \lambda^{*}\right)$ be a solution of (14), and from the vector function $F$ defined in (25), set

$$
\begin{aligned}
& G: \mathbb{R}^{n} \times \mathbb{R} \rightarrow \mathbb{R}^{n+1}, w^{*}=\left(x^{*}, \lambda^{*}\right) \mapsto G\left(w^{*}\right) \text { defined by } \\
& G\left(w^{*}\right)=G\left(x^{*}, \lambda^{*}\right)=\left[\begin{array}{c}
\lambda^{*} x^{*}-A x^{*} \\
\left\langle\mathbf{1}_{n}, x^{*}\right\rangle-1
\end{array}\right]
\end{aligned}
$$

and

$$
J=\Delta_{a}+\Delta_{b} G^{\prime}\left(w^{*}\right)
$$

where

$$
\begin{aligned}
& \Delta_{a}=\left[\begin{array}{ll}
I-V & 0 \\
0 & 0
\end{array}\right], \quad \Delta_{b}=\left[\begin{array}{ll}
V & 0 \\
0 & 1
\end{array}\right] \text { in the case of } \varphi_{\mathrm{min}}^{\mathrm{soc}} . \\
& \Delta_{a}=\left[\begin{array}{ll}
I-L_{w}^{-1} L_{x} & 0 \\
0 & 0
\end{array}\right], \Delta_{b}=\left[\begin{array}{ll}
I-L_{w}^{-1} L_{\left(\lambda^{*} x^{*}-A x^{*}\right)} & 0 \\
0 & 1
\end{array}\right] \text { in the case of } \varphi_{\mathrm{FB}}^{\mathrm{soc}}
\end{aligned}
$$

and $G^{\prime}\left(w^{*}\right)$ is given by

$$
G^{\prime}\left(w^{*}\right)=\left[\begin{array}{ll}
\lambda^{*} I-A & x^{*} \\
\mathbf{1}_{n}^{T} & 0
\end{array}\right] .
$$

Given a fixed solution $z^{*}=\left(x^{*}, y^{*}, \lambda^{*}\right)$ of (14) and let $w^{*}=\left(x^{*}, \lambda^{*}\right)$.

The point $w^{*}$ is said to be nondegenerate iff $w_{i}^{*}+G_{i}\left(w^{*}\right) \in \operatorname{int}\left(\mathcal{K}^{n_{i}}\right)$, for all $i \in$ $\{1, \ldots, r+1\}$. Then, the index sets

$$
\begin{aligned}
& \alpha:=\left\{i \in\{1, \ldots, r+1\}: w_{i}^{*} \in \operatorname{int}\left(\mathcal{K}^{n_{i}}\right), G_{i}\left(w^{*}\right)=0\right\}, \\
& \beta:=\left\{i \in\{1, \ldots, r+1\}: w_{i}^{*} \in \mathrm{bd}^{+}\left(\mathcal{K}^{n_{i}}\right), G_{i}\left(w^{*}\right) \in \mathrm{bd}^{+}\left(\mathcal{K}^{n_{i}}\right)\right\}, \\
& \gamma:=\left\{i \in\{1, \ldots, r+1\}: w_{i}^{*}=0, G_{i}\left(w^{*}\right) \in \operatorname{int}\left(\mathcal{K}^{n_{i}}\right)\right\} \text {, }
\end{aligned}
$$

where $\mathrm{bd}^{+}\left(\mathcal{K}^{n_{i}}\right)=\operatorname{bd}\left(\mathcal{K}^{n_{i}}\right) \backslash\{0\}$, form a partition of $\{1, \ldots, r+1\}$.

Moreover, we set

$$
\tilde{\Delta}_{a}:=\left[\begin{array}{ll}
I-\tilde{V} & 0 \\
0 & 0
\end{array}\right], \quad \tilde{\Delta}_{b}:=\left[\begin{array}{cc}
\tilde{V} & 0 \\
0 & 1
\end{array}\right], \quad \text { where } \tilde{V} \in \partial_{B} P_{\mathcal{K}}(\tilde{y})
$$

We note that $\lambda^{*} \tilde{\Delta}_{a}=\tilde{\Delta}_{\lambda^{*} a}$, and we set

$$
\tilde{J}=\tilde{\Delta}_{\lambda^{*} a}+\tilde{\Delta}_{b} G^{\prime}\left(w^{*}\right)
$$

Proposition 5.2 Let $z^{*}=\left(x^{*}, y^{*}, \lambda^{*}\right)$ be a fixed solution of $(14)$ with $w^{*}=\left(x^{*}, \lambda^{*}\right)$ being nondegenerate. If the submatrix $G^{\prime}\left(w^{*}\right)_{\alpha \alpha}$ is nonsingular and if its Schurcomplement

$$
G^{\prime}\left(w^{*}\right)_{\beta \beta}-G^{\prime}\left(w^{*}\right)_{\beta \alpha} G^{\prime}\left(w^{*}\right)_{\alpha \alpha}^{-1} G^{\prime}\left(w^{*}\right)_{\alpha \beta}
$$


is a P-matrix, then, the Jacobians $J$ and $\tilde{J}$, defined, respectively, in (31) and (35), are nonsingular matrices.

Proof Solving SOCEiCP is equivalent to solving the following system

$$
\begin{aligned}
& \text { find } w \in \mathbb{R}^{n+1} \backslash\{0\}, \text { such that } \\
& w \in \mathcal{K}, G(w) \in \mathcal{K},\langle w, G(w)\rangle=0,
\end{aligned}
$$

where $G$ is given by (30). The desired result follows immediately from Theorem 2.8 in [39].

Remark 5.1 If $r<n$, i.e., $\alpha \subset\{1, \ldots, n+1\}$, then $G^{\prime}\left(w^{*}\right)_{\alpha \alpha}$ is nonsingular if and only if $\left(\lambda^{*} I-A\right)_{\alpha \alpha}$ is nonsingular since in this case, we have

$G^{\prime}\left(w^{*}\right)_{\alpha \alpha}=\left(\lambda^{*} I-A\right)_{\alpha \alpha}$. Moreover, its Schur-complement is a P-matrix if and only if

$$
\left(\lambda^{*} I-A\right)_{\beta \beta}-\left(\lambda^{*} I-A\right)_{\beta \alpha}\left(\lambda^{*} I-A\right)_{\alpha \alpha}^{-1}\left(\lambda^{*} I-A\right)_{\alpha \beta}
$$

is a P-matrix.

Therefore, we get the following generalized theorems to the second-order cone.

Theorem 5.1 Assume that $z^{*}=\left(x^{*}, y^{*}, \lambda^{*}\right)$ is a fixed solution of (14) with $w^{*}=$ $\left(x^{*}, \lambda^{*}\right)$ nondegenerate. Then, all elements $H$ in the $B$-subdifferential $\partial_{B} \Phi\left(z^{*}\right)$, with $\varphi$ being one of the SOCC-functions $\varphi_{\mathrm{min}}^{\mathrm{soc}}$ or $\varphi_{\mathrm{FB}}^{\mathrm{soc}}$, are nonsingular if and only if $J$ is nonsingular.

Proof Let $H=H_{\min } \in \partial_{B} \Phi_{\min }\left(z^{*}\right)$ and let $X=(p, q, r) \in \mathbb{R}^{n} \times \mathbb{R}^{n} \times \mathbb{R}$ be an arbitrary vector such that

$$
H X=0_{\mathbb{R}^{2 n+1}} .
$$

The previous equation (37) can be written explicitly as

$$
\left[\begin{array}{lll}
I-V & V & 0 \\
\lambda^{*} I-A & -I & x^{*} \\
\mathbf{1}_{n}^{T} & 0 & 0
\end{array}\right]\left[\begin{array}{l}
p \\
q \\
r
\end{array}\right]=\left[\begin{array}{l}
0 \mathbb{R}^{n} \\
0_{\mathbb{R}^{n}} \\
0_{\mathbb{R}}
\end{array}\right]
$$

where $V \in \partial_{B} P_{\mathcal{K}^{n}}(x-y)$. Therefore,

$$
\begin{aligned}
(I-V) p+V q & =0 \mathbb{R}^{n}, \\
\left(\lambda^{*} I-A\right) p-q+r x^{*} & =0 \mathbb{R}^{n}, \\
\left\langle\mathbf{1}_{n}, p\right\rangle & =0 .
\end{aligned}
$$

Using (39), we have

$$
q=\left(\lambda^{*} I-A\right) p+r x^{*} .
$$

Consequently, (38) becomes

$$
(I-V) p+V\left(\lambda^{*} I-A\right) p+r V x^{*}=0 \mathbb{R}^{n} .
$$


Therefore,

$$
\begin{aligned}
& (I-V) p+V\left(\lambda^{*} I-A\right) p+r V x^{*}=0_{\mathbb{R}^{n}}, \\
& \left\langle\mathbf{1}_{n}, p\right\rangle=0
\end{aligned}
$$

which can be rewritten in the following matricial form

$$
\left[\begin{array}{ll}
I-V+V\left(\lambda^{*} I-A\right) & V x^{*} \\
\mathbf{1}_{n}^{T} & 0
\end{array}\right]\left[\begin{array}{l}
p \\
r
\end{array}\right]=\left[\begin{array}{l}
0_{\mathbb{R}^{n}} \\
0_{\mathbb{R}}
\end{array}\right] .
$$

By setting $Y=(p, r) \in \mathbb{R}^{n} \times \mathbb{R}$, clearly, (43) is equivalent to

$$
J Y=0_{\mathbb{R}^{n+1}} .
$$

Hence,

$$
H X=0_{\mathbb{R}^{2 n+1}} \Longleftrightarrow\left\{\begin{array}{l}
J Y=0_{\mathbb{R}^{n+1}}, \\
q=\left(\lambda^{*} I-A\right) p+r x^{*}
\end{array}\right.
$$

which completes the proof. For the case of the elements $H_{\mathrm{FB}} \in \partial_{B} \Phi_{\mathrm{FB}}\left(z^{*}\right)$, it is sufficient to apply the same steps given above.

Theorem 5.2 Assume that $\tilde{z}^{*}=\left(x^{*}, \tilde{y}^{*}, \lambda^{*}\right)$ is a fixed root of $\Phi_{\mathrm{LPM}}$ defined in (22) with $w^{*}=\left(x^{*}, \lambda^{*}\right)$ nondegenerate. Then the Jacobian $\tilde{J}$ defined in (35) is nonsingular if and only if all elements in $\partial_{B} \Phi_{\mathrm{LPM}}\left(\tilde{z}^{*}\right)$ are nonsingular.

Proof Let $\tilde{H} \in \partial_{B} \Phi_{\mathrm{LPM}}\left(\tilde{z}^{*}\right)$ and let $X=(p, q, r) \in \mathbb{R}^{n} \times \mathbb{R}^{n} \times \mathbb{R}$ such that

$$
\tilde{H} X=0_{\mathbb{R}^{2 n+1}} .
$$

The previous equation can be written explicitly as

$$
\left[\begin{array}{lll}
-\lambda^{*} I & \tilde{V} & -x^{*} \\
A & -I & 0 \\
\mathbf{1}_{n}^{T} & 0 & 0
\end{array}\right]\left[\begin{array}{l}
p \\
q \\
r
\end{array}\right]=\left[\begin{array}{l}
0_{\mathbb{R}^{n}} \\
0_{\mathbb{R}^{n}} \\
0_{\mathbb{R}}
\end{array}\right]
$$

where $\tilde{V} \in \partial_{B} P_{\mathcal{K}}(\tilde{y})$. Hence,

$$
\begin{aligned}
-\lambda^{*} p+\tilde{V} q-r x^{*} & =0 \mathbb{R}_{\mathbb{R}^{n}}, \\
A p-q & =0_{\mathbb{R}^{n}}, \\
\left\langle\mathbf{1}_{n}, p\right\rangle & =0 .
\end{aligned}
$$

Using (47), we have

$$
q=A p
$$


Consequently, (46) becomes

$$
\left(\lambda^{*} I-\tilde{V} A\right) p+r x^{*}=0 \mathbb{R}^{n}
$$

Therefore,

$$
\begin{aligned}
& \left(\lambda^{*} I-\tilde{V} A\right) p+r x^{*}=0 \mathbb{R}^{n}, \\
& \left\langle\mathbf{1}_{n}, p\right\rangle=0 .
\end{aligned}
$$

Note that

$$
I=I-\tilde{V}+\tilde{V}
$$

Then, (51) becomes

$$
\begin{aligned}
& \lambda^{*}(I-\tilde{V}) p+\tilde{V}\left(\lambda^{*} I-A\right) p+r x^{*}=0 \mathbb{R}_{\mathbb{R}^{n}}, \\
& \left\langle\mathbf{1}_{n}, p\right\rangle=0 .
\end{aligned}
$$

We note also that we have

$$
\tilde{V} x^{*}=x^{*} \text {. }
$$

Consequently, (52) can be rewritten in the following matricial form

$$
\left[\begin{array}{ll}
\lambda^{*}(I-\tilde{V})+\tilde{V}\left(\lambda^{*} I-A\right) & \tilde{V} x^{*} \\
\mathbf{1}_{n}^{T} & 0
\end{array}\right]\left[\begin{array}{l}
p \\
r
\end{array}\right]=\left[\begin{array}{l}
0_{\mathbb{R}^{n}} \\
0_{\mathbb{R}}
\end{array}\right] .
$$

By setting $Y=(p, r) \in \mathbb{R}^{n} \times \mathbb{R}$, it is clear that (53) is equivalent to

$$
\tilde{J} Y=0_{\mathbb{R}^{n+1}}
$$

Hence,

$$
\tilde{H} X=0_{\mathbb{R}^{2 n+1}} \Longleftrightarrow\left\{\begin{array}{l}
\tilde{J} Y=0_{\mathbb{R}^{n+1}} \\
q=A p
\end{array}\right.
$$

The desired conclusion of Theorem 5.2 follows immediately from the last equivalence.

\section{Numerical Experiments}

In this section, we apply the semismooth Newton method SNM, defined in Algorithm 1, in order to solve the SOCEiCP and give some numerical results to verify the efficiency of the LPM. The latter will be compared with two other solvers. The first one is the semismooth Newton method, related to $\varphi_{\min }^{\mathrm{soc}}$ defined in (16) (denoted by $\mathrm{SNM}_{\min }$ ), and the second one is related to $\varphi_{\mathrm{FB}}^{\mathrm{soc}}$ defined in (17) (denoted by $\mathrm{SNM}_{\mathrm{FB}}$ ). 
We choose the performance profiles developed in Dolan and Moré [24,25] as a tool to compare the solvers. Computing time, number of functions evaluations and number of failures are used as performance measures to compare the three solvers.

The numerical experiments are carried out in a Powerbook Mac OS 10.6.8 with a processor $2.33 \mathrm{GHz}$ Intel Core 2 Duo and 2Go memory. All the program codes are written and executed in Matlab 7.7.

Let $S$ be the set of the three solvers that will be compared. Before establishing the figures, we give some notations :

- $r_{p, s}$ denotes the performance ratio defined by

$$
r_{p, s}:=\frac{t_{p, s}}{\min \left\{t_{p, s}: s \in S\right\}},
$$

- $p$ is an element of the set of matrices $P=160$ taken to compare the solvers, with 10,000 initial points,

- $s$ denotes each solver,

- $n_{s}$ is the number of solvers,

- $t$ is a real factor, and

- $t_{p, s}$ denotes the average number of functions evaluations, the average computing time and the number of failures, respectively, in Figs. 1, 2, and 3.

Remark 6.1 In our case, a failure is declared if the Jacobian matrix is ill-conditioned or in the SNM defined in Algorithm 1, the number of iterations exceeded 100.

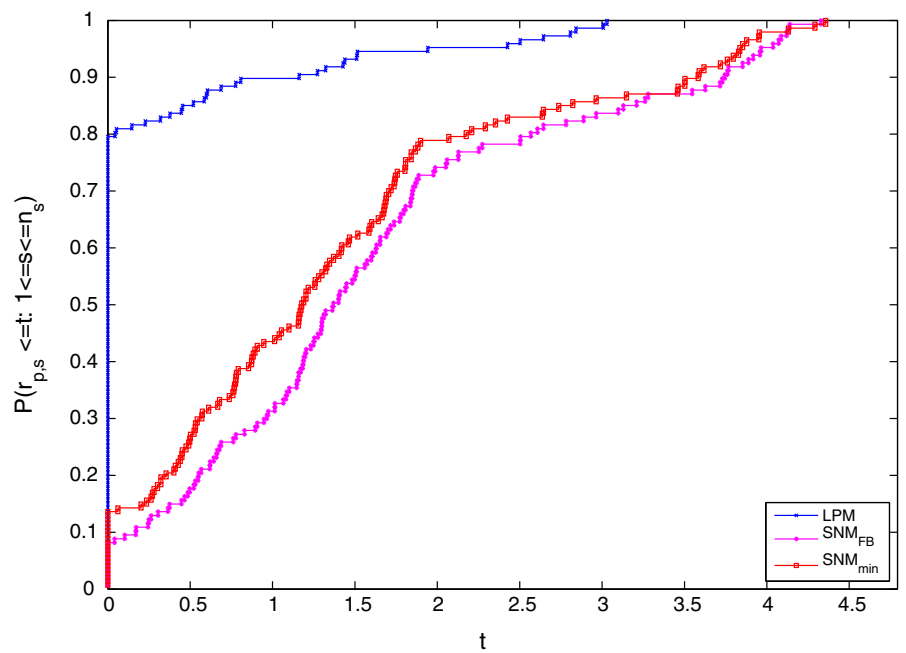

Fig. 1 Performance profiles where $t_{p, s}$ is the average number of functions evaluations 


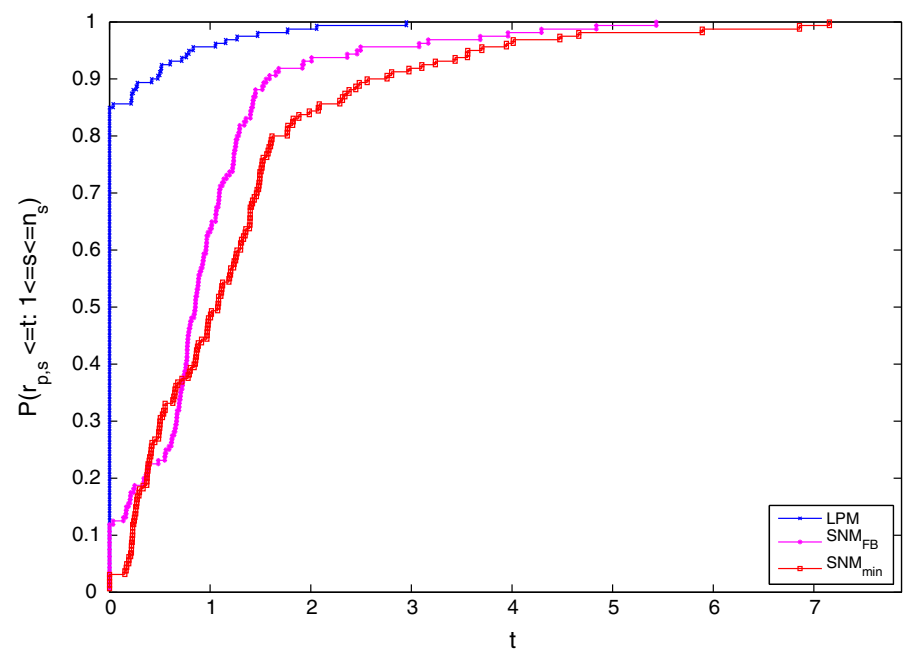

Fig. 2 Performance profiles where $t_{p, s}$ is the average computing time

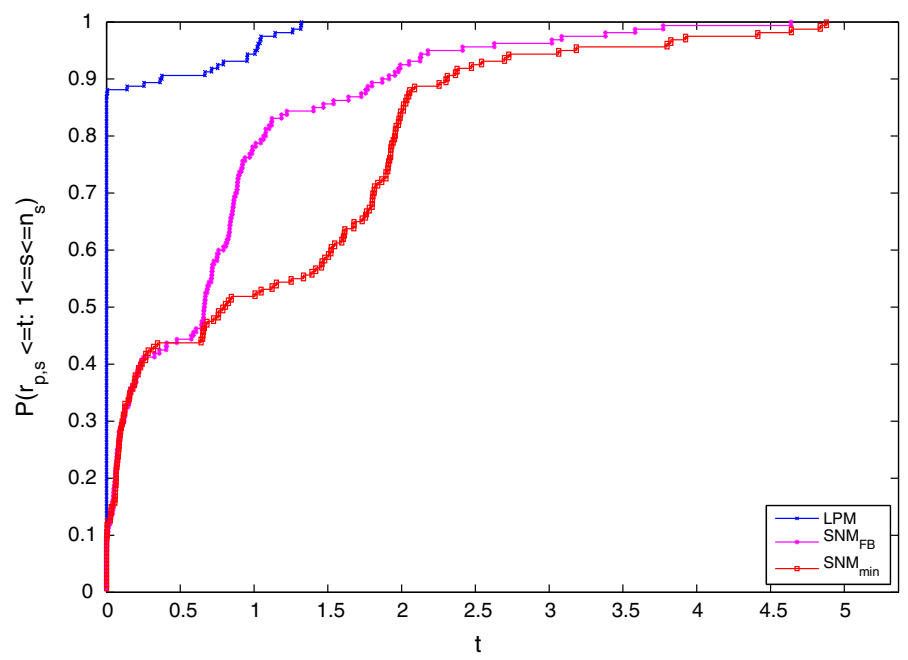

Fig. 3 Performance profiles where $t_{p, s}$ is the average number of failures

Figure 1 shows the performance profiles given by the three solvers, i.e., LPM, $\mathrm{SNM}_{\mathrm{FB}}$ and $\mathrm{SNM}_{\min }$. We compare the number of functions evaluations required to find a solution by each solver. Clearly, LPM has the most wins (since it has the highest probability). In the interval [0,0.5], LPM can solve $90 \%$ of the problems, while $\mathrm{SNM}_{\min }$ and $\mathrm{SNM}_{\mathrm{FB}}$ do not attain the $30 \%$. When $t \geq 2$, the performance of the latters becomes interesting.

In Fig. 2, the computing time is the comparing tool. Clearly, the performance profile of the LPM is interesting since it has the highest probability to solve problems. On the 
other hand, $\mathrm{SNM}_{\mathrm{FB}}$ and $\mathrm{SNM}_{\min }$ require a more running time to find a solution and have a lower number of wins.

Figure 3 shows the efficiency of the LPM, where the average number of failures to find each solution is the comparing tool. Clearly, in the interval [0, 0.5], LPM is able to solve about $90 \%$ of problems and detects the Lorentz eigenvalues while the others do not reach the $50 \%$. We see also that when $t \geq 2$, the performance of $\mathrm{SNM}_{\mathrm{FB}}$ becomes interesting. This allows us to conclude that the number of failures (in the sense of Remark 6.1) given by $\mathrm{SNM}_{\min }$ is high, which prevents to find solutions.

\section{Globally Convergent Methods}

In this section, we will discuss briefly a globalization of the three methods studied in the last sections by constructing appropriate merit functions associated to $\Phi_{\min }, \Phi_{\mathrm{FB}}$ and $\Phi_{\mathrm{LPM}}$ defined, respectively, in (14) and (22).

For the function $\Phi_{\mathrm{FB}}$, we introduce the following classical merit function $\Psi_{\mathrm{FB}}$ : $\mathbb{R}^{2 n+1} \rightarrow \mathbb{R}^{2 n+1}$, defined by

$$
\Psi_{\mathrm{FB}}(z):=\frac{1}{2} \Phi_{\mathrm{FB}}(z)^{T} \Phi_{\mathrm{FB}}(z) .
$$

We note that the function $\Psi_{\mathrm{FB}}$ is of class $C^{1}$ and that SOCEiCP is also equivalent to the following unconstrained global optimization problem

$$
\min _{z \in \mathbb{R}^{2 n+1}} \Psi_{\mathrm{FB}}(z)
$$

Since $\Psi_{\mathrm{FB}}$ is continuously differentiable, it is easy to force global convergence of the algorithm by using the gradient of the merit function (see [40]).

\section{Algorithm 2 (Semismooth Newton Method SNM)}

Step 0. (Data)

Choose parameters $\delta, \rho \in] 0,1[, \sigma \in] 0,0.5[, \kappa>2$ and initial point $z^{0}:=\left(x^{0}, y^{0}, \lambda^{0}\right) \in \mathbb{R}^{n} \times \mathbb{R}^{n} \times \mathbb{R}$. Give the error bound $0<\epsilon<<1$ and set $k=0$. Step 1. (Termination criteria)

If $\left\|\nabla \Psi_{F B}\left(z^{k}\right)\right\| \leq \epsilon$, stop, where the function $\Psi_{\mathrm{FB}}(\cdot)$ is defined in (55).

Step 2. (Search direction calculation)

At a current point $z^{k}$, we select $M^{k} \in \partial \Phi_{\mathrm{FB}}\left(z^{k}\right)$ and compute $h^{k}$ by solving the linear system

$$
M^{k} h^{k}=-\Phi_{\mathrm{FB}}\left(z^{k}\right) .
$$

If system (56) is unsolvable or if $h^{k}$ does not satisfy the following condition

$$
\nabla \Psi_{F B}\left(z^{k}\right)^{T} h^{k} \leq-\rho\left\|h^{k}\right\|^{\kappa},
$$

then set

$$
h^{k}=-\nabla \Psi_{F B}\left(z^{k}\right) .
$$


Step 3. (Line search)

Let $m_{k}$ be the smallest non-negative integer $m$ such that

$$
\Psi_{F B}\left(z^{k}+\delta^{m} h^{k}\right)-\Psi_{F B}\left(z^{k}\right) \leq \sigma \delta^{m} \nabla \Psi_{F B}\left(z^{k}\right)^{T} h^{k},
$$

and let $\alpha_{k}:=\delta^{m_{k}}$ and $z^{k+1}:=z^{k}+\alpha_{k} h^{k}$.

Step 4. (Update)

Set $k:=k+1$ and go to Step 1 .

We have the following convergence result (see [40] for more details).

Theorem 7.1 [40] Let $F$ be a $C^{1}$-function and let $\left\{z^{k}=\left(x^{k}, y^{k}, \lambda^{k}\right)\right\}$ denote a sequence generated by Algorithm 2. Then, the following statements hold.

(a) Each accumulation point of the sequence $\left\{z^{k}\right\}$ is a stationary point of $\Psi_{\mathrm{FB}}$.

(b) If the sequence $\left\{z^{k}\right\}$ has an accumulation point $z^{*}$ which is a BD-regular solution of the system $\Phi_{\mathrm{FB}}(z)=0$, then $\left\{z^{k}\right\}$ converges $Q$-superlinealry to $z^{*}$.

(c) If, in addition to the assumptions of part $(b)$, the gradient of $F$ is locally Lipshitz continuous at $z^{*}$, then $\left\{z^{k}\right\}$ converges $Q$-quadratically to $z^{*}$.

Remark 7.1 We can construct also merit functions $\Psi_{\min }$ and $\Psi_{\mathrm{LPM}}$ associated to the natural residual function $\Phi_{\min }$ and $\Phi_{\text {LPM }}$ defined, respectively, in (14) and (22):

$$
\Psi_{\min }(z)=\frac{1}{2}\left\|\Phi_{\min }(z)\right\|^{2} \text { and } \Psi_{\mathrm{LPM}}(\tilde{z})=\frac{1}{2}\left\|\Phi_{\mathrm{LPM}}(\tilde{z})\right\|^{2} .
$$

The drawback of these two functions are their non-differentiability. As an immediate consequence, numerical methods based on the gradient of the function, such as steepest descent method and Newton's method, cannot be applicable directly to $\Psi_{\min }$ and $\Psi_{\mathrm{LPM}}$. To overcome this difficulty, Fukushima et al. [13] and Hayashi et al. [19] proposed smoothing and regularization methods by replacing the original non differentiable function by a sequence of differentiable approximations. The boundedness of the levelsets (or the coercivity) of the objective function plays an important role to ensure the global convergence of a descent method. Further investigations must be done in this direction for SOCEiCP.

\section{Conclusions}

In this paper, we have studied numerical methods for solving the second-order cone eigenvalue complementarity problem. We have reformulated such a problem into a system of semismooth equations. Moreover, we have extended the LPM, introduced in [1], to solve the SOCEiCP. The performance profiles highlight the efficiency of the LPM method for solving SOCEiCP. It will be interesting to compare the globalization of the three methods in the future (using remark 7.1). Another natural question is to generalize the LPM to the mixed eigenvalue complementarity problems (where only some components of the vector $x \in \mathbb{R}^{n}$ are cone-constrained and the identity $I$ in (3) is replaced by some matrix of order $n$ ). This kind of problems appears in 
the study of the equilibrium state of mechanical systems with unilateral contact [8]. Another interesting question would be the generalization of the LPM method for solving quadratic eigenvalue complementarity problems. This is out of the scope of this paper and will probably be the subject of a future project of research.

\section{References}

1. Adly, S., Rammal, H.: A new method for solving eigenvalue complementarity problems. Comput. Optim. Appl. 55, 703-731 (2013)

2. Adly, S., Seeger, A.: A nonsmooth algorithm for cone-constrained eigenvalue problems. Comput. Optim. Appl. 49, 299-318 (2011)

3. Seeger, A., Vicente-Perez, J.: On cardinality of Pareto spectra. Electron. J. Linear Algebra 22, 758-766 (2011)

4. Martins, J.A.C., Barbarin, S., Raous, M., Pinto da Costa, A.: Dynamic stability of finite dimensional linearly elastic systems with unilateral contact and coulomb friction. Comput. Methods Appl. Mech. Eng. 177, 289-328 (1999)

5. Martins, J.A.C., Pinto da Costa, A.: Stability of finite-dimensional nonlinear elastic systems with unilateral contact and friction. Int. J. Solids Struct. 37, 2519-2564 (2000)

6. Martins, J.A.C., Pinto da Costa, A.: Bifurcations and instabilities in frictional contact problems: theoretical relations, computational methods and numerical results. In: Neittaanmäki, P., Rossi, T., Korotov, S., Oñate, E., Periaux, J., Knörzer, D. (eds.) Fourth European Congress on Computational Methods in Applied Sciences and Engineering (ECCOMAS 2004). Jyväskylä, Finland, 24-28 July. Complete article in CD-ROM, vol. II. ISBN 951-39-1869-6 (2004)

7. Martins, J.A.C., Pinto da Costa, A.: Computation of bifurcations and instabilities in some frictional contact problems. In: Second European Conference on Computational Mechanics (ECCM 2001). Cracow, Poland, 26-29 June. Complete article in CD-ROM (2001)

8. Martins, J.A.C., Pinto da Costa, A., Figueiredo, I.N., Júdice, J.J.: The directional instability problem in systems with frictional contacts. Comput. Methods Appl. Mech. Eng. 193, 357-384 (2004)

9. Júdice, J.J., Raydan, M., Rosa, S.S., Santos, S.A.: On the solution of the symmetric eigenvalue complementarity problem by the spectral projected gradient algorithm. Numer. Algorithms 47, 391-407 (2008)

10. Pindo da Costa, A., Seeger, A.: Cone-constrained eigenvalue problems: theory and algorithms. Comput. Optim. Appl. 45(1), 25-57 (2008)

11. Dirkse, S.P., Ferris, M.C.: The PATH solver: a non-monotone stabilization scheme for mixed complementarity problems. Optim. Methods Softw. 5, 123-156 (1995)

12. Lobo, M.S., Vandenberghe, L., Boyd, S., Lebret, H.: Applications of second-order cone programming. Linear Algebra Appl. 284, 193-228 (1998)

13. Fukushima, M., Luo, Z.-Q., Tseng, P.: Smoothing functions for second-order cone complementarity problems. SIAM J. Optim. 12, 436-460 (2001)

14. Chen, X.D., Sun, D., Sun, J.: Complementarity functions and numerical experiments for second-order cone complementarity problems. Comput. Optim. Appl. 25, 39-56 (2003)

15. Chen, J.-S., Chen, X., Tseng, P.: Analysis of nonsmooth vector-valued functions associated with secondorder cones. Math. Program. 101, 95-117 (2004)

16. Bonnans, J.F., Ramírez, H.: Perturbation analysis of second-order cone programming problems. Math. Program. 104, 205-227 (2005)

17. Chen, J.-S.: Alternative proofs for some results of vector-valued functions associated with second-order cones. J. Nonlinear Convex. Anal. 6, 297-325 (2005)

18. Chen, J.-S., Tseng, P.: An unconstrained smooth minimization reformulation of the second-order cone complementarity problem. Math. Program. 104, 293-327 (2005)

19. Hayashi, S., Yamashita, N., Fukushima, M.: A combined smoothing and regularization method for monotone second-order cone complementarity problems. SIAM J. Optim. 15(2), 593-615 (2005) 
20. Kato, H., Fukushima, M.: An SQP-type algorithm for nonlinear second-order cone programs. Optim. Lett. 1, 129-144 (2007)

21. Sun, D., Sun, J.: Strong semismoothness of the Fischer-Burmeister SDC and SOC complementarity functions. Math. Program. 103, 575-581 (2005)

22. Tseng, P.: Smoothing methods for second-order cone programs/complementarity problems. Talk presented at the SIAM Conference on Optimization, Stockholm (May 2005)

23. Yamashita, H., Yabe, H.: A primal-dual interior point method for nonlinear optimization over second order cones. Optim. Methods Softw. 24(3), 407-426 (2009)

24. Cops: See http://mcs.anl.gov/more/cops/

25. Dolan, E.D., Moré, J.J.: Benchmarking optimization software with performance profiles. Math. Program. Ser. A 91, 201-213 (2002)

26. Faraut, J., Korányi, A.: Analysis on Symmetric Cones. Oxford Mathematical Monographs, Oxford University Press, New York (1994)

27. Koecher, M.: The Minnesota Notes on Jordan Algebras and Their Applications. Edited and annotated by Brieg, A., Walcher, S., Berlin (1999)

28. Júdice, J.J., Sherali, H., Ribeiro, I.M., Rosa, S.: On the asymmetric eigenvalue complementarity problem. Optim. Methods Softw. 24, 549-586 (2009)

29. Alizadeh, F., Goldfarb, D.: Second-order cone programming. Math. Program. 95(1), 3-51 (2003)

30. Clarke, F.H.: Optimization and Nonsmooth Analysis. Wiley, New York (1983), reprinted by SIAM, Philadelphia, PA (1990)

31. Mifflin, R.: Semismooth and semiconvex functions in constrained optimization. SIAM J. Control Optim. 15, 957-972 (1977)

32. Qi, L., Sun, J.: A nonsmooth version of Newton's method. Math. Program. 58, 353-367 (1993)

33. Kanzow, C., Ferenczi, I., Fukushima, M.: On the local convergence of semismooth Newton methods for linear and nonlinear second-order cone programs without strict complementarity. SIAM J. Optim. 20(1), 297-320 (2009)

34. Pang, J.-S., Sun, D., Sun, J.: Semismooth homeomorphisms and strong stability of semidefinite and Lorentz cone complementarity problems. Math. Oper. Res. 28, 39-63 (2003)

35. Seeger, A., Torki, M.: On eigenvalues induced by a cone constraint. Linear Algebra Appl. 372, 181-206 (2003)

36. Gowda, M.S., Sznajder, R., Tao, J.: P-properties for linear transformations on Euclidean Jordan algebras. Linear Algebra Appl. 393, 203-232 (2004)

37. Kong, L., Tunel, L., Xiu, N.: Fischer-Burmeister complementarity function on Euclidean Jordan algebras. Pac. J. Optim. 6(2), 423-440 (2007)

38. Pan, S., Chen, J.-S.: A damped Gauss-Newton method for the second-order cone complementarity problem. Appl. Math. Optim. 59, 293-318 (2009)

39. Kanzow, C., Kleinmichel, H.: A new class of semismooth Newton type methods for nonlinear complementarity problems. J. Comput. Optim. Appl. 11, 227-251 (1998)

40. Facchinei, F., Fischer, A., Kanzow, C.: A semismooth Newton type method for variational inequalities: the case of box constraints. In: Ferris, M.C., Pang, J.S. (eds.) Complement. Var. Probl. State Art, pp. 76-90. Pennsylvania, SIAM, Philadelphia (1997) 